\title{
Kripke semantics for fuzzy logics
}

\section{Parvin Safari \& Saeed Salehi}

\section{Soft Computing}

A Fusion of Foundations,

Methodologies and Applications

ISSN 1432-7643

Volume 22

Number 3

Soft Comput (2018) 22:839-844

DOI 10.1007/s00500-016-2387-4

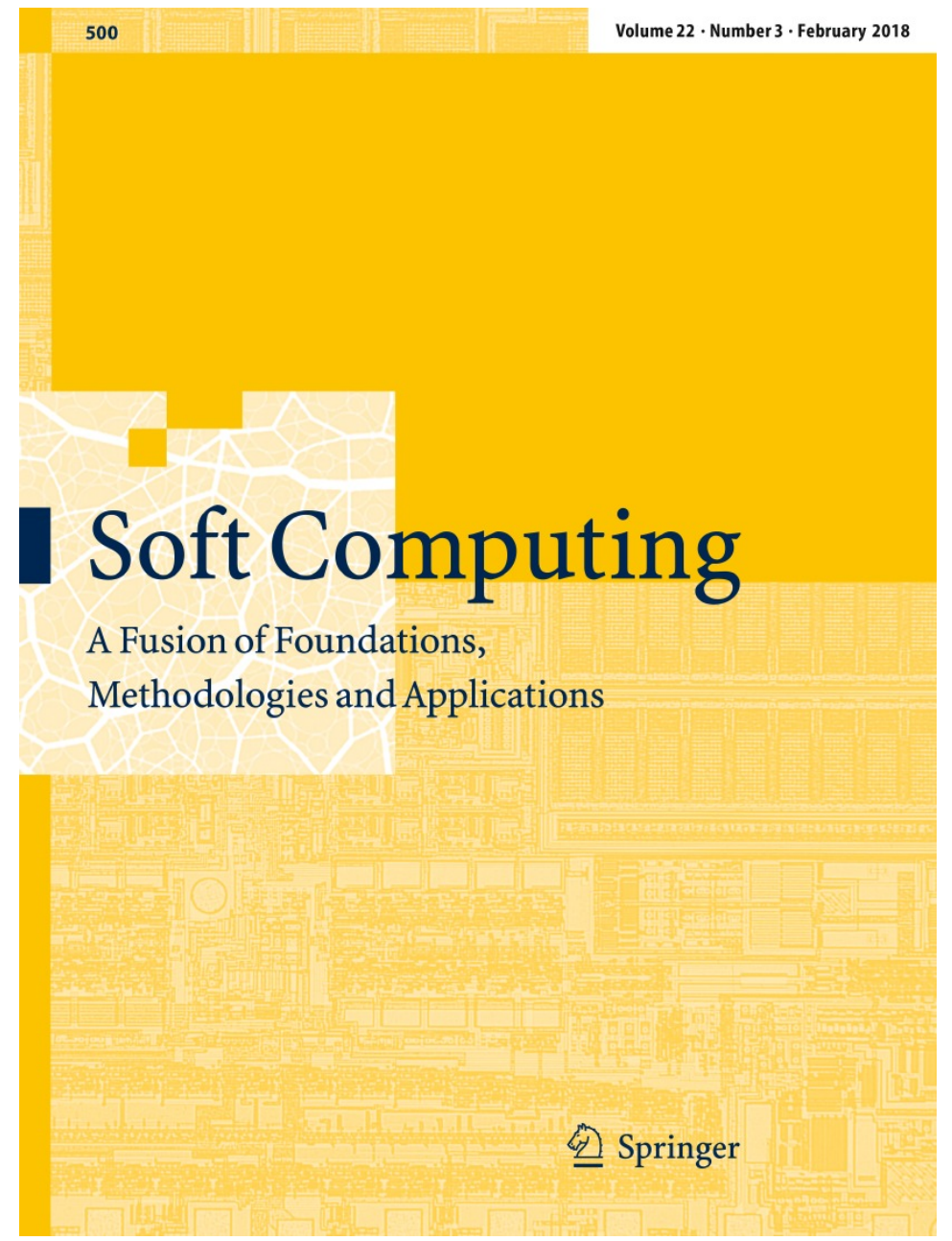

Springer 
Your article is protected by copyright and all rights are held exclusively by SpringerVerlag Berlin Heidelberg. This e-offprint is for personal use only and shall not be selfarchived in electronic repositories. If you wish to self-archive your article, please use the accepted manuscript version for posting on your own website. You may further deposit the accepted manuscript version in any repository, provided it is only made publicly available 12 months after official publication or later and provided acknowledgement is given to the original source of publication and a link is inserted to the published article on Springer's website. The link must be accompanied by the following text: "The final publication is available at link.springer.com". 


\title{
Kripke semantics for fuzzy logics
}

\author{
Parvin Safari $^{1}$ - Saeed Salehi ${ }^{1}$
}

Published online: 12 October 2016

(C) Springer-Verlag Berlin Heidelberg 2016

\begin{abstract}
Kripke frames (and models) provide a suitable semantics for sub-classical logics; for example, intuitionistic logic (of Brouwer and Heyting) axiomatizes the reflexive and transitive Kripke frames (with persistent satisfaction relations), and the basic logic (of Visser) axiomatizes transitive Kripke frames (with persistent satisfaction relations). Here, we investigate whether Kripke frames/models could provide a semantics for fuzzy logics. For each axiom of the basic fuzzy logic, necessary and sufficient conditions are sought for Kripke frames/models which satisfy them. It turns out that the only fuzzy logics (logics containing the basic fuzzy logic) which are sound and complete with respect to a class of Kripke frames/models are the extensions of the Gödel logic (or the super-intuitionistic logic of Dummett); indeed this logic is sound and strongly complete with respect to reflexive, transitive and connected (linear) Kripke frames (with persistent satisfaction relations). This provides a semantic characterization for the Gödel logic among (propositional) fuzzy logics.
\end{abstract}

Keywords Fuzzy logics · The basic fuzzy logic · Gödel logic . Dummett logic - Kripke frames · Soundness . Completeness $\cdot$ Semantics

Communicated by V. Loia.

This a part of the Ph.D. thesis of the first author written under the supervision of the second author at the University of Tabriz.

Saeed Salehi

salehipour@tabrizu.ac.ir

http://saeedsalehi.ir

Parvin Safari

p_safari@tabrizu.ac.ir

1 Department of Mathematics, University of Tabriz, 29 Bahman Blvd., P.O. Box 51666-17766, Tabriz, Iran

\section{Introduction and preliminaries}

Kripke frames provide a semantics for modal logics and for some sub-classical logics such as intuitionistic logic (of Brouwer and Heyting) and basic logic (of Visser). Visser basic logic is sound and strongly complete with respect to transitive Kripke frames (Visser 1981), and the intuitionistic logic is sound and strongly complete with respect to reflexive and transitive Kripke frames (Mints 2002). It could be expected that a class of Kripke frames could provide a suitable semantics for the basic fuzzy logic (introduced in Hájek 1998). For each axiom of this logic, all the Kripke frames/models that satisfy it will be investigated. We shall see that the only (fuzzy) logics which contain the basic fuzzy logic and are sound and strongly complete with respect to a class of Kripke frames/models are extensions of the Gödel logic, or equivalently the Dummett logic (cf. Dummett 1959; Bendová 1999). This logic can be aximatized as the intuitionistic logic plus the axiom $(\varphi \rightarrow \psi) \vee(\psi \rightarrow \varphi)$ and is sound and strongly complete with respect to reflexive, transitive, and connected Kripke frames (with persistent satisfaction relations).

Definition 1 (Kripke frames) A Kripke frame is a directed graph, i.e., an ordered pair $\langle K, R\rangle$ where $R \subseteq K^{2}$ is a binary relation on $K$. In a Kripke frame $\langle K, R\rangle$, the members of $K$ are called nodes and the relation $R$ is called the accessability relation; if $k R k^{\prime}$, then the node $k^{\prime}$ is said to be accessible from the node $k$.

Definition 2 (Reflexivity and transitivity) A binary relation $R \subseteq K \times K$ is

- reflexive, when for any $k \in K, k R k$ holds.

- transitive, when for any $k, k^{\prime}, k^{\prime \prime} \in K$, if $k R k^{\prime}$ and $k^{\prime} R k^{\prime \prime}$ hold, then $k R k^{\prime \prime}$ holds. 
A Kripke frame is called reflexive/transitive, when the relation $R$ is so.

Definition 3 (Transitive closure) For $R \subseteq K \times K$ and $k \in$ $K$, let $R^{1}[k]=R[k]=\{x \in K \mid k R x\}$ be the image of $\{k\}$ under $R$, and let $R^{2}[k]=\{x \in K \mid \exists y \in K(k R y R x)\}$, and generally for any $n \in \mathbb{N}$ let

$R^{n}[k]=\left\{x \in K \mid \exists y_{1}, \ldots, y_{n-1} \in K\left(k R y_{1} R y_{2} R \cdots R y_{n-1} R x\right)\right\}$.

The transitive closure of $R$ on $\{k\}$ is then $R^{+}[k]=$ $\bigcup_{n=1}^{\infty} R^{n}[k]$. Define also $R^{++}[k]=\bigcup_{n=2}^{\infty} R^{n}[k]$.

Definition 4 (Connectedness) A relation $R \subseteq K \times K$ is called connected, when for any $k \in K$ and any $k^{\prime}, k^{\prime \prime} \in R^{+}[k]$, either $k^{\prime} R k^{\prime \prime}$ or $k^{\prime \prime} R k^{\prime}$ holds (cf. Švejdar and Bendová 2000).

Definition 5 (Syntax of fuzzy logic) Formulas of Propositional fuzzy logic are built from the constant $\Perp$ (for the falsity) and the connectives $\&, \rightarrow$ (for conjunction and implication) together with a countably infinite set of atoms, denoted Atoms.

Let us note that then the negation of a formula $\varphi$ becomes $\varphi \rightarrow \Perp \Perp$ in this language.

Definition 6 (Kripke models) A Kripke model is a triple $\mathscr{K}=\langle K, R, \models\rangle$ where $\langle K, R\rangle$ is a Kripke frame and $\vDash \subseteq K \times$ Atoms is a satisfaction relation. The satisfaction relation can be extended to all the (propositional) formulas, i.e., to $\vDash \subseteq K \times$ Formulas, as follows (Formulas is the set of all formulas):

- No node satisfies $\Perp$, i.e., $k \not \models \Perp$ for all $k \in K$.

- The conjunction is satisfied if and only if each component is satisfied, i.e.,

$k \vDash(\varphi \& \psi) \Longleftrightarrow k \vDash \varphi$ and $k \vDash \psi$.

- The implication is satisfied if and only if whenever an accessible node satisfies the antecedent then it also satisfies the consequent, i.e.,

$$
\begin{aligned}
k \vDash & (\varphi \rightarrow \psi) \\
& \Longleftrightarrow \text { for all } k^{\prime} \in K\left(\text { if } k R k^{\prime} \text { and } k^{\prime} \vDash \varphi \text { then } k^{\prime} \vDash \psi\right) \\
& \Longleftrightarrow \forall k^{\prime} \in R[k]\left(k^{\prime} \vDash \varphi \longrightarrow k^{\prime} \vDash \psi\right) .
\end{aligned}
$$

Remark 1 (Truth) The formula $\Perp \rightarrow-\Perp$ is always true and holds in every node of any Kripke model (by definition). Let us denote it by $\bar{\pi}(=\Perp \rightarrow \Perp)$.

Definition 7 (Satisfaction) A formula is satisfied in a Kripke model when it is satisfied in every node of that model. A Kripke frame satisfies a formula when every Kripke model with that frame satisfies the formula. A rule is said to be satisfied in a Kripke model when the satisfaction of the premise(s) of the rule in a node implies the satisfaction of its conclusion in that node. A rule is said to be satisfied in a Kripke frame when it is satisfied in every Kripke model with that frame.

Definition 8 (Persistency) A satisfaction relation $\vDash \subseteq K \times$ Atoms is called to be (atom) persistent with respect to $R \subseteq$ $K \times K$ (cf. Švejdar and Bendová 2000) when for any $k, k^{\prime} \in K$ and $p \in$ Atoms, if $k \vDash p$ and $k R k^{\prime}$, then $k^{\prime} \vDash p$; this property is called atom persistency. A satisfaction relation $\models \subseteq K \times$ Formulas is called to be (formula) persistent with respect to $R \subseteq K \times K$ when for any $k, k^{\prime} \in K$ and $\varphi \in$ Formulas, if $k \vDash \varphi$ and $k R k^{\prime}$, then $k^{\prime} \vDash \varphi$; this property is called formula persistency.

Convention The restriction of a relation $S \subseteq A \times B$ to a subset $C \subseteq A$ is denoted by $\left.S\right|_{C}$, i.e., $\left.S\right|_{C}=S \cap(C \times B)$.

Proposition 1 (Atom/formula persistency) In a Kripke mo$\operatorname{del}\langle K, R, \models\rangle$ if the restriction of $R$ to $R^{+}[k]$, i.e., $\left.R\right|_{R^{+}[k]}$, is transitive for some node $k \in K$, then the atom persistency in (every node of) $R^{+}[k]$ implies the formula persistency (in $\left.R^{+}[k]\right)$.

Proof By induction on the formula $\varphi$, we show that for every $k^{\prime}, k^{\prime \prime} \in R^{+}[k]$ if $k^{\prime} R k^{\prime \prime}$ and $k^{\prime} \vDash \varphi$, then $k^{\prime \prime} \vDash \varphi$ :

- For atomic formula $\varphi$, we have $k^{\prime \prime} \vDash \varphi$ by the assumption (also by definition, $k^{\prime \prime} \not \models \Perp$ always holds).

- For $\varphi=\psi \& \theta$ ( $\psi$ and $\theta$ are formulas) by definition, $k^{\prime} \vDash \psi$ and $k^{\prime} \vDash \theta$, so by the induction hypothesis $k^{\prime \prime} \vDash \psi$ and $k^{\prime \prime} \vDash \theta$, whence, $k^{\prime \prime} \vDash \psi \& \theta$ holds.

- For $\varphi=\psi \rightarrow \theta$, we show that $k^{\prime \prime} \vDash \psi \rightarrow \theta$ which is equivalent to

$\forall k^{\prime \prime \prime} \in R\left[k^{\prime \prime}\right]\left(k^{\prime \prime \prime} \vDash \psi \Longrightarrow k^{\prime \prime \prime} \vDash \theta\right)$.

So, let us assume that (1) $\left.R\right|_{R^{+}[k]}$ is transitive, (2) $k^{\prime} \vDash$ $\psi \rightarrow \theta$, (3) $k^{\prime \prime \prime} \vDash \psi$, and (4) $k^{\prime} R k^{\prime \prime} R K^{\prime \prime \prime}$ for $k^{\prime}, k^{\prime \prime}, k^{\prime \prime \prime} \in$ $R^{+}[k]$. By (1) and (4), we have $k^{\prime} R k^{\prime \prime \prime}$, and so by (2) and (3), $k^{\prime \prime \prime} \vDash \theta$ holds.

Lemma 1 (Transitivity lemma) In a Kripke frame $\langle K, R\rangle$, if $R$ is reflexive and for all $k \in K$, the restriction of $R$ to $R^{+}[k]$, i.e., $\left.R\right|_{R^{+}[k]}$, is transitive, then $R$ is transitive.

Proof If $R$ were not transitive, there would exist some $k_{1}, k_{2}, k_{3} \in K$ such that $k_{1} R k_{2}$ and $k_{2} R k_{3}$ but $k_{1} R k_{3}$. Now, trivially, $k_{2}, k_{3} \in R^{+}\left[k_{1}\right]$ and by the reflexivity of $R$ we also have $k_{1} \in R^{+}\left[k_{1}\right]$. But then $\left.R\right|_{R^{+}\left[k_{1}\right]}$ is not transitive, contradiction! 


\subsection{The basic fuzzy logic}

The axiom of basic logic (BL) are (cf. Hájek 1998)

$$
\begin{aligned}
& \left(A_{1}\right) \quad(\varphi \rightarrow \psi) \rightarrow[(\psi \rightarrow \theta) \rightarrow(\varphi \rightarrow \theta)] \\
& \left(A_{2}\right) \quad(\varphi \& \psi) \rightarrow \varphi \\
& \left(A_{3}\right) \quad(\varphi \& \psi) \rightarrow(\psi \& \varphi) \\
& \left(A_{4}\right) \quad(\varphi \&[\varphi \rightarrow \psi]) \rightarrow(\psi \&[\psi \rightarrow \varphi]) \\
& \left(A_{5} a\right)[\varphi \rightarrow(\psi \rightarrow \theta)] \rightarrow[(\varphi \& \psi) \rightarrow \theta] \\
& \left(A_{5} b\right)[(\varphi \& \psi) \rightarrow \theta] \rightarrow[\varphi \rightarrow(\psi \rightarrow \theta)] \\
& \left(A_{6}\right)[(\varphi \rightarrow \psi) \rightarrow \theta] \rightarrow[([\psi \rightarrow \varphi] \rightarrow \theta) \rightarrow \theta] \\
& \left(A_{7}\right) \stackrel{\Perp}{\Perp} \rightarrow \varphi
\end{aligned}
$$

and its (only) rule is Modus Ponens

$$
\text { (MP) } \frac{A, \quad A \rightarrow B}{B} \text {. }
$$

\section{Basic fuzzy logic and Kripke frames/models}

It immediately follows from the definitions that

Proposition 2 (Universality of $A_{2}, A_{3}, A_{7}$, and $\varphi \rightarrow \varphi \& \varphi$ ) The axioms $\left(A_{2}\right),\left(A_{3}\right),\left(A_{7}\right)$, and also $\varphi \rightarrow(\varphi \& \varphi)$ are satisfied in every Kripke frame.

It can also be easily checked that the Modus Ponens $(M P)$ rule is satisfied in every reflexive Kripke frame. The converse is also true (cf. Celani and Jansana 2001, Proposition 5.1).

Theorem 1 (MP and reflexivity) The only rule of the basic fuzzy logic (MP) is satisfied in a Kripke frame $\langle K, R\rangle$ if and only if $R$ is reflexive.

Proof If the relation $R$ is reflexive then for any node $k \in K$ we have $k \vDash \varphi, k \vDash \varphi \rightarrow \psi \Longrightarrow k \vDash \psi$ just because $k R k$. Now, if $R$ is not reflexive then there exists some $k \in K$ such that $k \not R k$. For atoms $p, q$ let $\vDash$ be $(K \times\{p\}) \cup(R[k] \times\{q\})$. Then $k \vDash p$ and $k \vDash p \rightarrow q$ because for any $k^{\prime}$ with $k R k^{\prime}$ we have $k^{\prime} \vDash q$. But $k \not \models q$ because $k \notin R[k]$. So, the rule (MP) is not satisfied at node $k$.

The axiom $\left(A_{1}\right)$ is satisfied in every transitive Kripke frame. The following theorem characterizes exactly the frames in which this axiom is satisfied.

Theorem 2 ( $A_{1}$ and transitivity) The axiom $\left(A_{1}\right)$ is satisfied in a Kripke frame $\langle K, R\rangle$ if and only if $\left.R\right|_{R^{+}[k]}$ is transitive for all $k \in K$.

Proof Fix a $k \in K$ and suppose that $\left.R\right|_{R^{+}[k]}$ is transitive. We show $k \vDash\left(A_{1}\right)$, or equivalently $\forall k^{\prime} \in R[k]\left(k^{\prime} \vDash(\varphi \rightarrow\right.$ $\left.\psi) \Longrightarrow k^{\prime} \vDash[(\psi \rightarrow \theta) \rightarrow(\varphi \rightarrow \theta)]\right)$. That is equivalent to showing, for a fixed $k^{\prime} \in R[k]$, that $\forall k^{\prime \prime} \in R\left[k^{\prime}\right]\left(k^{\prime \prime} \vDash \psi \rightarrow\right.$ $\left.\theta \Longrightarrow k^{\prime \prime} \vDash \varphi \rightarrow \theta\right)$, assuming $k^{\prime} \vDash(\varphi \rightarrow \psi)$, and this is in turn equivalent to showing, assuming $k^{\prime \prime} \vDash \psi \rightarrow \theta$ for a fixed $k^{\prime \prime} \in R\left[k^{\prime}\right]$, that $\forall k^{\prime \prime \prime} \in R\left[k^{\prime \prime}\right]\left(k^{\prime \prime \prime} \vDash \varphi \Longrightarrow k^{\prime \prime \prime} \vDash \theta\right)$. Thus, let us assume that (1) $\left.R\right|_{R^{+}[k]}$ is transitive and $k R k^{\prime} R k^{\prime \prime} R k^{\prime \prime \prime}$, (2) $k^{\prime} \vDash \varphi \rightarrow \psi$, (3) $k^{\prime \prime} \vDash \psi \rightarrow \theta$, and (4) $k^{\prime \prime \prime} \vDash \varphi$. We then show that $k^{\prime \prime \prime} \vDash \theta$ : By (1), since $k^{\prime \prime \prime}, k^{\prime \prime}, k^{\prime} \in R^{+}[k]$, we have $k^{\prime} R k^{\prime \prime \prime}$ and so by (2) and (4) we can infer that $k^{\prime \prime \prime} \vDash \psi$. Whence, (3) implies that $k^{\prime \prime \prime} \vDash \theta$ holds.

So, the if part of the theorem has been proved. For the only if part, assume that for a node $k_{0} \in K$, in a Kripke frame $\langle K, R\rangle$, the relation $\left.R\right|_{R^{+}\left[k_{0}\right]}$ is not transitive; i.e., there are $k_{1}, k_{2}, k_{3} \in R^{+}\left[k_{0}\right]$ such that $k_{1} R k_{2} R k_{3}$ but $k_{1} \not R k_{3}$. For atoms $p, q, r$ let the satisfaction relation $\vDash$ be $(K \times\{p\}) \cup\left(R\left[k_{1}\right] \times\{q\}\right) \cup\left(\left(R\left[k_{1}\right] \cap R\left[k_{2}\right]\right) \times\{r\}\right)$. Since we have $k_{1}, k_{2}, k_{3} \in R^{+}\left[k_{0}\right]$, there are $\ell_{1}, \ldots, \ell_{n} \in K$ (for some $n \geqslant 0$ ) such that $k_{0} R \ell_{1} R \cdots R \ell_{n} R k_{1} R k_{2} R k_{3}$ (when $n=0$, then $\left.\ell_{n}=k_{0}\right)$. We now show that the instance $(p \rightarrow q) \rightarrow[(q \rightarrow r) \rightarrow(p \rightarrow r)]$ of $\left(A_{1}\right)$ is not satisfied at $\ell_{n}$. To see this, we note that $k_{2} \not \models p \rightarrow r$, because $k_{2} R k_{3}, k_{3} \vDash p$ but $k_{3} \not \models r$ for $k_{3} \notin R\left[k_{1}\right]$, and $k_{2} \vDash q \rightarrow r$ because for any $k \in K$ if $k_{2} R k \vDash q$, then $k \in R\left[k_{2}\right]$ and $k \in R\left[k_{1}\right]$ so $k \vDash r$. Hence, we conclude that $k_{1} \not \models(q \rightarrow r) \rightarrow(p \rightarrow r)$, but $k_{1} \vDash p \rightarrow q$ because for any $k \in K$ if $k_{1} R k \vDash p$, then $k \in R\left[k_{1}\right]$ and so $k \vDash q$. Thus, $\ell_{n} \not \models(p \rightarrow q) \rightarrow[(q \rightarrow r) \rightarrow(p \rightarrow r)]$.

It can be seen that the axiom $\left(A_{4}\right)$ is satisfied in every reflexive Kripke model whose satisfaction relation is (formula) persistent (with respect to the accessibility relation). Here, we give an exact characterizations for all the Kripke models which satisfy this axiom.

Theorem 3 ( $A_{4}$ and reflexivity + Persistency) The axiom $\left(A_{4}\right)$ is satisfied in every Kripke model $\langle K, R, \models\rangle$ in which for every $k \in K$ the restricted relation $\left.R\right|_{R^{+}[k]}$ is reflexive and $\vDash \mid R_{R^{+}[k]}$ is formula persistent with respect to $R$. Conversely, if $\left(A_{4}\right)$ is satisfied in a Kripke frame then for all $k \in K$ the relation $\left.R\right|_{R^{+}[k]}$ is reflexive and the restriction of the satisfaction relations to the sets $R^{+}[k]$ (for every $k \in K$ ) on those frames should be formula persistent with respect to $R$.

Proof For a fixed Kripke model $\langle K, R, \models\rangle$ and fixed node $k \in K$, suppose that $\left.R\right|_{R^{+}[k]}$ is reflexive and that $\left.\vDash\right|_{R^{+}[k]}$ has the formula persistency property. We show that $k \vDash\left(A_{4}\right)$ or equivalently $\forall k^{\prime} \in R[k]\left(k^{\prime} \vDash \varphi \&[\varphi \rightarrow \psi] \Longrightarrow k^{\prime} \vDash\right.$ $\psi \&[\psi \rightarrow \varphi])$. Thus, it suffices to show that $k^{\prime} \vDash \psi$ and $\forall k^{\prime \prime} \in R\left[k^{\prime}\right]\left(k^{\prime \prime} \vDash \psi \Longrightarrow k^{\prime \prime} \vDash \varphi\right)$, if $k R k^{\prime} \vDash \varphi \&[\varphi \rightarrow$ $\psi]$. Whence, we assume that (1) $\left.R\right|_{R^{+}[k]}$ is reflexive and $k R k^{\prime} R k^{\prime \prime}$, (2) $k^{\prime} \vDash \varphi \&[\varphi \rightarrow \psi]$, (3) $k^{\prime \prime} \vDash \psi$, and (4) $\left.\vDash\right|_{R^{+}[k]}$ is formula persistent; and show that $k^{\prime} \vDash \psi$ and $k^{\prime \prime} \vDash \varphi$. By (2) we have (5) $k^{\prime} \vDash \varphi$ and (6) $k^{\prime} \vDash \varphi \rightarrow \psi$. So, by (4) and (1) we also have $k^{\prime \prime} \vDash \varphi$. By (1) again, we have $k^{\prime} R k^{\prime}$ which by (5) and (6) implies that $k^{\prime} \vDash \psi$ holds.

Now, we suppose that the axiom $\left(A_{4}\right)$ is satisfied in a Kripke frame $\langle K, R\rangle$, and show that for any $k \in K$ the relation $\left.R\right|_{R^{+}[k]}$ is reflexive. If $\left.R\right|_{R^{+}\left[k_{0}\right]}$ is not reflexive for some 
$k_{0} \in K$, then there are $\ell_{1}, \ldots, \ell_{n} \in K(n \geqslant 0)$ such that $k_{0} R \ell_{1} R \cdots R \ell_{n} R k_{1} R k_{1}$. Define the satisfaction relation $\vDash$ to be $\left\langle k_{1}, p\right\rangle$ for some atom $p$. We show that under this satisfaction relation the instance $(p \&[p \rightarrow q]) \rightarrow(q \&[q \rightarrow p])$ of $\left(A_{4}\right)$ is not satisfied at $\ell_{n}$. That is because $k_{1} \vDash p \&(p \rightarrow q)$ by definition and the fact that for no $k \in R\left[k_{1}\right]$ we can have $k \vDash p$ (by $\left.k_{1} R k_{1}\right)$. On the other hand by definition $k_{1} \not \models q$ and so $k_{1} \not \models q \&(q \rightarrow p)$.

Next, if $\left.\vDash\right|_{R^{+}\left[k_{0}\right]}$ is not formula persistent with respect to $R$ in a Kripke model $\langle K, R, \models\rangle$ and node $k_{0} \in K$, then there are two nodes $k_{1}, k_{2} \in R^{+}\left[k_{0}\right]$ and a formula $\varphi$ such that $k_{1} R k_{2}$ and $k_{1} \vDash \varphi$ but $k_{2} \not \models \varphi$. Also there are some $\ell_{1}, \ldots, \ell_{n} \in K(n \geqslant 0)$ such that $k_{0} R \ell_{1} R \cdots R \ell_{n} R k_{1}$. We show that the instance $(\varphi \&[\varphi \rightarrow \bar{\pi}]) \rightarrow(\bar{\pi} \&[\bar{\pi} \rightarrow \varphi])$ of $\left(A_{4}\right)$ (see Remark 1 for the definition of $\bar{\pi}$ ) is not satisfied in $\langle K, R, \vDash\rangle$ at $\ell_{n}$ : because at $k_{1}$ (for which $\ell_{n} R k_{1}$ holds) we have $k_{1} \vDash \varphi \&[\varphi \rightarrow \bar{\pi}$. (since $k \vDash \bar{\pi}$ holds for any $k$ ) but $k_{1} \not \models \bar{\pi} \rightarrow \varphi$ since for $k_{2} \in R\left[k_{1}\right]$ we have $k_{2} \not \models \varphi$ (and of course $k_{2} \vDash \bar{\pi}$ ).

The axiom $\left(A_{5} a\right)$, too, is satisfied in every reflexive frame. Here is an exact characterization.

Theorem 4 ( $A_{5} a$ and reflexivity) The axiom $\left(A_{5} a\right)$ is satisfied in a Kripke frame $\langle K, R\rangle$ if and only if $\left.R\right|_{R^{2}[k]}$ is reflexive for all $k \in K$.

Proof Fix a node $k \in K$ in a Kripke frame $\langle K, R\rangle$ for which $\left.R\right|_{R^{2}[k]}$ is reflexive. For showing $k \vDash\left(A_{5} a\right)$, we show that $\forall k^{\prime} \in R[k]\left(k^{\prime} \vDash \varphi \rightarrow(\psi \rightarrow \theta) \Longrightarrow k^{\prime} \vDash(\varphi \& \psi) \rightarrow \theta\right)$, which is equivalent to $\forall k^{\prime \prime} \in R\left[k^{\prime}\right]\left(k^{\prime \prime} \vDash(\varphi \& \psi) \Longrightarrow k^{\prime \prime} \vDash\right.$ $\theta)$, for some fixed $k^{\prime} \in R[k]$ with $k^{\prime} \vDash \varphi \rightarrow(\psi \rightarrow \theta)$. Whence, we assume that (1) $\left.R\right|_{R^{2}[k]}$ is reflexive, (2) $k^{\prime} \vDash$ $\varphi \rightarrow(\psi \rightarrow \theta)$, (3) $k^{\prime \prime} \vDash \varphi \& \psi$ and (4) $k R k^{\prime} R k^{\prime \prime}$, and show that $k^{\prime \prime} \vDash \theta$ : by (3) we have (5) $k^{\prime \prime} \vDash \psi$; the assumptions (2) and (4) imply that (6) $k^{\prime \prime} \vDash \psi \rightarrow \theta$. By the reflexivity of $\left.R\right|_{R^{2}[k]}$ and $k^{\prime \prime} \in R^{2}[k]$ we have $k^{\prime \prime} R k^{\prime \prime}$, and so it follows from (5) and (6) that $k^{\prime \prime} \vDash \theta$ holds. This proves the if part of the theorem.

For the converse, the only if part, assume that for a node $k_{0} \in K$ in a Kripke frame $\langle K, R\rangle$, the restricted relation $\left.R\right|_{R^{2}\left[k_{0}\right]}$ is not reflexive; i.e., there is $k \in R^{2}\left[k_{0}\right]$ such that $k R k$. Let us note that for some $k^{\prime}$ we have $k_{0} R k^{\prime} R k$. Let $\vDash$ be $(\{k\} \times\{p, q\})$ for atoms $p, q, r$. We show that the instance $[p \rightarrow(q \rightarrow r)] \rightarrow[(p \& q) \rightarrow r]$ of $\left(A_{5} a\right)$ is not satisfied at $k_{0}$ : we have $k^{\prime} \not \models(p \& q) \rightarrow r$ because at $k \in R\left[k^{\prime}\right]$ we have $k \vDash p \& q$ but $k \not \models r$. On the other hand $k^{\prime} \vDash p \rightarrow(q \rightarrow r)$ because for any $\ell \in R\left[k^{\prime}\right]$ if $\ell \vDash p$, then $\ell=k$ but then $k \vDash q \rightarrow r$ since no node in $R[k]$ satisfies $q$ (note that $k \notin R[k]$ ). Concluding, it follows that $k_{0} \not \models[p \rightarrow(q \rightarrow r)] \rightarrow[(p \& q) \rightarrow r]$.

Similarly, we provide an exact characterizations for Kripke models which satisfy the axiom $\left(A_{5} b\right)$.
Theorem 5 ( $A_{5} b$ and transitivity + persistency) The axiom $\left(A_{5} b\right)$ is satisfied in every Kripke frame $\langle K, R\rangle$ in which for all $k \in K$ the relation $\left.R\right|_{R^{+}[k]}$ is transitive and $\left.\vDash\right|_{R^{++}[k]}$ is formula persistent with respect to $R$. Conversely, if $\left(A_{5} b\right)$ is satisfied in a Kripke frame $\langle K, R\rangle$, then for all $k \in K$ the relation $\left.R\right|_{R^{+}[k]}$ is transitive and the restriction of the satisfaction relations to the sets $R^{++}[k]$ (for every $k \in K$ ) on that frame should be formula persistent with respect to $R$.

Proof For a Kripke model $\langle K, R, \models\rangle$ and a node $k \in K$ of it, if $\left.R\right|_{R^{+}[k]}$ is transitive and $\left.\vDash\right|_{R^{++}[k]}$ is formula persistent with respect to $R$, then we show that $k \vDash\left(A_{5} b\right)$ which is equivalent to $\forall k^{\prime} \in R[k]\left(k^{\prime} \vDash(\varphi \& \psi) \rightarrow \theta \Longrightarrow k^{\prime} \vDash \varphi \rightarrow(\psi \rightarrow \theta)\right)$ or equivalent to $\forall k^{\prime \prime} \in R\left[k^{\prime}\right]\left(k^{\prime \prime} \vDash \varphi \Longrightarrow k^{\prime \prime} \vDash \psi \rightarrow \theta\right)$, under the assumption $k R k^{\prime} \vDash(\varphi \& \psi) \rightarrow \theta$. This, in turn, is equivalent to $\forall k^{\prime \prime \prime} \in R\left[k^{\prime \prime}\right]\left(k^{\prime \prime \prime} \vDash \psi \Longrightarrow k^{\prime \prime \prime} \vDash \theta\right)$ assuming that $k^{\prime} R k^{\prime \prime} \vDash \varphi$. Whence, we assume that (1) the relation $\vDash$ $\left.\right|_{R^{++}[k]}$ is atom persistent with respect to $R,(2)$ the restricted relation $\left.R\right|_{R^{+}[k]}$ is transitive and we have that $k R k^{\prime} R k^{\prime \prime} R k^{\prime \prime \prime}$, (3) $k^{\prime} \vDash(\varphi \& \psi) \rightarrow \theta$, (4) $k^{\prime \prime} \vDash \varphi$ and (5) $k^{\prime \prime \prime} \vDash \psi$; and show that $k^{\prime \prime \prime} \vDash \theta$ : from (1), (4) and (5), noting that $k^{\prime \prime}, k^{\prime \prime \prime} \in$ $R^{++}[k]$, we have (6) $k^{\prime \prime \prime} \vDash \varphi \& \psi$. Then from (2) we have $k^{\prime} R k^{\prime \prime \prime}$ and so (3) and (6) imply that $k^{\prime \prime \prime} \vDash \theta$ holds.

Now, if for a node $k_{0} \in K$ in a Kripke frame $\langle K, R\rangle$ the restricted relation $\left.R\right|_{R^{+}\left[k_{0}\right]}$ is not transitive, then there are $k_{1}, k_{2}, k_{3} \in R^{+}\left[k_{0}\right]$ such that $k_{1} R k_{2} R k_{3}$, but $k_{1} R k_{3}$. Also, there are $\ell_{1}, \ldots, \ell_{n} \in K(n \geqslant 0)$ such that $k_{0} R \ell_{1} R \cdots R \ell_{n} R k_{1}$. Let the satisfaction relation $\vDash$ be defined as $\left(R\left[k_{1}\right] \times\{r\}\right) \cup$ $\left\{\left\langle k_{2}, p\right\rangle\right\} \cup\left\{\left\langle k_{3}, q\right\rangle\right\}$ for some atoms $p, q, r$. Now we show that the instance $[(p \& q) \rightarrow r] \rightarrow[p \rightarrow(q \rightarrow r)]$ of $\left(A_{5} b\right)$ is not satisfied at $\ell_{n}$ : we have $k_{1} \vDash p \& q \rightarrow r$ because for no $k \in R\left[k_{1}\right]$ can we have $k \vDash p \& q$. Also, $k_{2} \not \models q \rightarrow r$ because at $k_{3} \in R\left[k_{2}\right]$ we have $k_{3} \vDash q$ but $k_{3} \not \models r$ (notice that $k_{3} \notin R\left[k_{1}\right]$ ), therefore, $k_{1} \not \models p \rightarrow(q \rightarrow r)$ because at $k_{2} \in R\left[k_{1}\right]$ we have $k_{2} \vDash p$ but $k_{2} \not \models q \rightarrow r$. Now that we have $k_{1} \vDash p \& q \rightarrow r$ and $k_{1} \not \models p \rightarrow(q \rightarrow r)$ we therefore infer the desired conclusion $\ell_{n} \not \models[(p \& q) \rightarrow r] \rightarrow[p \rightarrow(q \rightarrow r)]$.

Finally, if for a node $k_{0} \in K$ in a Kripke model $\langle K, R, \models\rangle$ the restricted satisfaction relation $\left.\vDash\right|_{R^{++}\left[k_{0}\right]}$ is not formula persistent (with respect to $R$ ), then there exist two nodes $k_{1}, k_{2} \in R^{++}\left[k_{0}\right]$ and a formula $\varphi$ such that $k_{1} R k_{2}, k_{1} \vDash \varphi$ and $k_{2} \not \models \varphi$. Also, by Definition 3, there are $\ell_{1}, \ldots, \ell_{n} \in K(n \geqslant 1)$ such that $k_{0} R \ell_{1} R \cdots R \ell_{n} R k_{1}$. We show that the instance $[(\varphi \& \bar{\pi}) \rightarrow \varphi] \rightarrow[\varphi \rightarrow(\bar{\pi} \rightarrow \varphi)]$ of $\left(A_{5} b\right)$ (see Remark 1 for the definition of $\overline{\mathrm{T}}$ ) is not satisfied in this model at $\ell_{n-1}$; let us recall that if $n=1$, then $\ell_{n-1}=k_{0}$. To see this, firstly, we note that for $\ell_{n} \in R\left[\ell_{n-1}\right]$ we have $\ell_{n} \vDash(\varphi \& \bar{\pi}) \rightarrow \varphi$ (indeed $k \vDash(\varphi \& \bar{\pi}) \rightarrow \varphi$ holds for any node $k$ ). Secondly, $\ell_{n} \not \models \varphi \rightarrow(\bar{\pi} \rightarrow \varphi)$ because for $k_{1} \in R\left[\ell_{n}\right]$ we have $k_{1} \vDash \varphi$ but $k_{1} \not \models \pi \rightarrow \varphi$ since at $k_{2} \in R\left[k_{1}\right]$ we have (of course $k_{2} \vDash \bar{\pi}$ and also) $k_{2} \not \models \varphi$.

Let us pause for a moment and see where we have got from these results so far. By Proposition 2, the axioms $\left(A_{2}\right)$, 
$\left(A_{3}\right)$ and $\left(A_{7}\right)$ (and also Gödel's Axiom $\varphi \rightarrow \varphi \& \varphi$ ) are satisfied in all Kripke frames. By Theorem 1, only reflexive Kripke frames can satisfy the $(M P)$ rule. By Theorem 2 the axiom $\left(A_{1}\right)$ can be satisfied in a Kripke frame $\langle K, R\rangle$ if and only if $\left.R\right|_{R^{+}[k]}$ is transitive, for all $k \in K$. So, suitable Kripke frames for fuzzy logics should be reflexive and transitive by Lemma 1. Moreover, the satisfaction relations on those (reflexive and transitive) Kripke frames should be (formula) persistent by Theorem 3, since Kripke models on those frames should satisfy the axiom $\left(A_{4}\right)$ as well; Theorem 4 (for the axiom $A_{5} a$ ) and Theorem 5 (for the axiom $A_{5} b$ ) confirm this even more. So, one should necessarily consider reflexive, transitive and persistent Kripke models for fuzzy logics.

Unfortunately, we have been unable to find a good characterizations for Kripke frame/models which satisfy the axiom $\left(A_{6}\right)$. One candidate for a class of Kripke frames which satisfy this axiom is the class of connected (Definition 4) Kripke frames. Indeed, $\left(A_{6}\right)$ is satisfied in every (persistent and) connected Kripke model (see Theorem 6 below). But the converse does not hold: the Kripke model $\langle\{\emptyset,\{a\},\{b\}\}, \subseteq, \emptyset\rangle$ (with the empty satisfaction relation) is reflexive, transitive and persistent but not connected (assuming $a \neq b$ ), while it satisfies $\left(A_{6}\right)$ and every classical tautology. Below (in Theorem 6), we show that if a reflexive and transitive Kripke frame satisfies $\left(A_{6}\right)$ with persistent satisfaction relations, then it must be connected.

Before proving Theorem 6 let us make a little note about the linearity axiom $(\varphi \rightarrow \psi) \vee(\psi \rightarrow \varphi)$ which, over the (propositional) intuitionistic logic, axiomatizes the Kripke frames whose accessibility relations are linear orders. The logic resulted by appending this axiom to the intuitionistic logic is called Dummett logic (see Dummett 1959 and the Conclusions below).

Lemma 2 (The connectedness axiom) The formula $(\varphi \rightarrow$ $\psi) \vee(\psi \rightarrow \varphi)$ is satisfied in all (formula) persistent and connected Kripke models.

Proof For formulas $\varphi$, $\psi$, if $k \not \models(\varphi \rightarrow \psi) \vee(\psi \rightarrow \varphi)$, then there exist $k^{\prime}, k^{\prime \prime} \in R[k]$ such that $k^{\prime} \vDash \varphi$ but $k^{\prime} \not \models \psi$, and $k^{\prime \prime} \vDash \psi$ but $k^{\prime \prime} \not \models \varphi$. By connectedness (and $k^{\prime}, k^{\prime \prime} \in$ $\left.R^{+}[k]\right)$ we have either $k^{\prime} R k^{\prime \prime}$ or $k^{\prime \prime} R k^{\prime}$. Now, if $k^{\prime} R k^{\prime \prime}$, then from $k^{\prime} \vDash \varphi$ we will have $k^{\prime \prime} \vDash \varphi$ by (formula) persistency; a contradiction (since $k^{\prime \prime} \not \models \varphi$ ). Similarly, a contradiction follows from $k^{\prime \prime} R k^{\prime}$.

Theorem 6 ( $A_{6}$ and connectedness, by reflexivity, transitivity and persistency) The axiom $\left(A_{6}\right)$ is satisfied in every connected and persistent Kripke model. Also, if a reflexive and transitive Kripke frame satisfies $\left(A_{6}\right)$ with persistent satisfaction relations, then it must be connected.

Proof Suppose $\langle K, R, \models\rangle$ is connected and persistent. For a node $k \in K$, and formulas $\varphi, \psi, \theta$, we show that $k \vDash[(\varphi \rightarrow$ $\psi) \rightarrow \theta] \rightarrow[([\psi \rightarrow \varphi] \rightarrow \theta) \rightarrow \theta]$. This is equivalent to $\forall k^{\prime} \in R[k]\left(k^{\prime} \vDash(\varphi \rightarrow \psi) \rightarrow \theta \Longrightarrow k^{\prime} \vDash([\psi \rightarrow \varphi] \rightarrow\right.$ $\theta) \rightarrow \theta)$. So, fix a $k^{\prime} \in R[k]$ with $k^{\prime} \vDash(\varphi \rightarrow \psi) \rightarrow \theta$; we prove that $\forall k^{\prime \prime} \in R\left[k^{\prime}\right]\left(k^{\prime \prime} \vDash[(\psi \rightarrow \varphi) \rightarrow \theta] \Longrightarrow k^{\prime \prime} \vDash \theta\right)$. Whence, we assume that (1) $R$ is connected and $k R k^{\prime} R k^{\prime \prime}$, $(2) \vDash$ is formula persistent with respect to $R$, (3) $k^{\prime} \vDash(\varphi \rightarrow$ $\psi) \rightarrow \theta$, and (4) $k^{\prime \prime} \vDash(\psi \rightarrow \varphi) \rightarrow \theta$; and show that $k^{\prime \prime} \vDash \theta$. By Lemma 2 we have either (i) $k^{\prime \prime} \vDash \varphi \rightarrow \psi$ or (ii) $k^{\prime \prime} \vDash \psi \rightarrow \varphi$. In case of (i), from (1) and (3) we already infer that $k^{\prime \prime} \vDash \theta$. In case of (ii), we note that $k^{\prime \prime} R k^{\prime \prime}$ by (1) (and that the connectedness of $R$ implies the reflexivity of $\left.R\right|_{R^{+}[k]}$ ) and so from (4) we can conclude that $k^{\prime \prime} \vDash \theta$.

Now, assume (for the sake of contradiction) that the Kripke frame $\langle K, R\rangle$ is reflexive and transitive but not connected. Then there must exist some nodes $k, k^{\prime}, k^{\prime \prime} \in K$ such that $k R k^{\prime}, k R k^{\prime \prime}, k^{\prime} R k^{\prime \prime}$ and $k^{\prime \prime} R k^{\prime}$. Let us already note that then $k \notin R\left[k^{\prime}\right] \cup R\left[k^{\prime \prime}\right]$ and $k^{\prime} \notin R\left[k^{\prime \prime}\right]$ also $k^{\prime \prime} \notin R\left[k^{\prime}\right]$. For atoms $p, q, r$, define the satisfaction relation $\vDash$ on this frame to be $\left(R\left[k^{\prime}\right] \times\{p\}\right) \cup\left(R\left[k^{\prime \prime}\right] \times\{q\}\right) \cup([R[k] \cap\{\ell \in K \mid \ell \not R k\}] \times\{r\})$. By the transitivity of $R$, this satisfaction relation is atom persistent (since, e.g., if $\ell \vDash r$ and $\ell R \ell^{\prime}$, then from $k R \ell$ and $\ell R k$, and the transitivity of $R$, we have $k R \ell^{\prime}$ and also $\ell^{\prime} R k$ since otherwise if $\ell^{\prime} R k$, then from $\ell R \ell^{\prime}$, and the transitivity of $R$, we would have $\ell R k$ contradiction); thus $\vDash$ is formula persistent (by the transitivity of $R$ and Proposition 1 ). We show that under this satisfaction relation the instance $[(p \rightarrow$ $q) \rightarrow r] \rightarrow[([q \rightarrow p] \rightarrow r) \rightarrow r]$ of $\left(A_{6}\right)$ is not satisfied at $k$. We firstly note that $k \not \models p \rightarrow q$ (because at $k^{\prime} \in R[k]$ we have $k^{\prime} \vDash p$ and $k^{\prime} \not \nvdash q$ ) and also $k \not \models q \rightarrow p$ (because at $k^{\prime \prime} \in R[k]$ we have $k^{\prime \prime} \vDash q$ and $k^{\prime \prime} \not \models p$ ), and secondly that $k \vDash(p \rightarrow q) \rightarrow r$ and $k \vDash(q \rightarrow p) \rightarrow r$ (because for any $\ell \in R[k]$ if $\ell \vDash p \rightarrow q$ or $\ell \vDash q \rightarrow p$ then, by the persistency, $\ell \not R k$ and so $\ell \vDash r)$. Finally, $k \not \models([q \rightarrow p] \rightarrow r) \rightarrow r$ since $k \vDash[q \rightarrow p] \rightarrow r$ but $k \not \models r$.

Finally, the main result of the paper is the following which follows from all the previous results:

Corollary 1 (Kripke models for the basic fuzzy logic) $A$ Kripke model satisfies the axioms (and the rule) of the basic fuzzy logic if and only if it is reflexive, transitive, and connected, and the satisfaction relation is (formula) persistent with respect to the accessibility relation.

This can indeed be seen as a negative result in the theory of Kripke models, since it shows that no class of Kripke frames can axiomatize exactly BL or the fuzzy logics that do not contain Gödel logic. But it has also some positive sides discussed in the next section.

\section{Conclusions}

Gödel fuzzy logic is axiomatized as BL plus the axiom $\varphi \rightarrow(\varphi \& \varphi)$ of idempotence of conjunction (cf. Bendová 
1999). Dummett (1959) showed that this logic can be completely axiomatized by the axioms of intuitionistic logic plus the axiom $(\varphi \rightarrow \psi) \vee(\psi \rightarrow \varphi)$. Indeed, the GödelDummett logic is sound and strongly complete with respect to reflexive, transitive, connected, and persistent Kripke models. In Corollary 1, we showed that the only class of Kripke models which could be sound and (strongly) complete for a logic containing BL must contain the class of reflexive, transitive, connected and persistent Kripke models. In the other words, any logic that contains BL and is axiomatizing a class of Kripke frames/models must also contain the Gödel-Dummett logic (cf. Proposition 2). So, a Kripkemodel-theoretic characterization of Gödel fuzzy logic is that it is the smallest fuzzy logic containing the basic fuzzy logic which is sound and complete with respect to a class of Kripke frames/models. Also, the class of reflexive, transitive, connected, and persistent Kripke models is the smallest class that can be axiomatized by a propositional fuzzy logic.

\section{Compliance with ethical standards}

Conflict of interest The authors declare that they have no conflict of interest.

Human and animal rights statement This article does not contains any studies with human participants or animals performed by any of the authors.

\section{References}

Bendová K (1999) A note on Gödel fuzzy logic. Soft Comput 2:167. doi:10.1007/s005000050049

Celani S, Jansana R (2001) A closer look at some subintuitionistic logics. Notre Dame J Form Logic 42:225-255. doi:10.1305/ndjfl/ 1063372244

Dummett M (1959) A propositional calculus with denumerable matrix. J Symb Logic 24:97-106. doi:10.2307/2964753

Hájek P (1998) Metamathematics of fuzzy logic. Kluwer, Dordrecht. ISBN: 9781402003707. doi:10.1007/978-94-011-5300-3

Mints G (2002) A short introduction to intuitionistic logic. Kluwer, Dordrecht. ISBN: 9780306463945. doi:10.1007/b115304

Švejdar V, Bendová K (2000) On inter-expressibility of logical connectives in Gödel fuzzy logic. Soft Comput 4:103-105. doi:10.1007/ s005000000036

Visser A (1981) A propositional logic with explicit fixed points. Stud Log 40:155-175. doi:10.1007/BF01874706 